\title{
Edge Effects and the Population Structure of Humboldt Bay, California, Eelgrass (Zostera marina L.)
}

\author{
Joshua S. Neely \\ School of Biological Sciences, Washington State University, Pullman, WA 99164, USA \\ Correspondence should be addressed to Joshua S. Neely; joshua.neely@ashford.edu
}

Received 31 July 2014; Revised 20 October 2014; Accepted 3 November 2014; Published 23 November 2014

Academic Editor: Daniel I. Rubenstein

Copyright ( 2014 Joshua S. Neely. This is an open access article distributed under the Creative Commons Attribution License, which permits unrestricted use, distribution, and reproduction in any medium, provided the original work is properly cited.

\begin{abstract}
The physical structure of a habitat (e.g., fragmentation) may affect the distribution of genetic diversity within a population, and genetic diversity may alter ecological function. This study investigates the population genetics of Humboldt Bay, California, eelgrass in light of the habitat's fragmented physical structure. Historical and ongoing dredging operations in the bay maintain channels between fragmented tidal mudflats that are inhabited by eelgrass. A sample of 469 individuals, from 11 discrete fragments, was genotyped using microsatellite analysis. Ramets were collected at multiple points located at the edge and in the interior of each fragment. The sampling scheme was designed to detect evidence of population structure within and between fragments, as well as between edge and interior bed positions. Genetic diversity is not shown to differ among fragments and is not consistent with widespread clonality. Heterozygosity levels indicate that Hardy-Weinberg equilibrium predominates across loci in most instances. Comparisons of edge and interior bed positions also reveal similarities between positions for the metrics described above. Results suggest efficient gene flow between fragments and between bed positions. Such findings, which indicate the unstructured nature of the population, can guide resource managers in making evolutionarily informed conservation decisions by discouraging assumptions of low genetic diversity and insignificant sexual reproduction.
\end{abstract}

\section{Introduction}

One of the most scrutinized ecological aspects of habitat fragmentation is the effect that habitat edges exert on the biotic community. Traditionally, such studies have focused on forest edges and their effect on animal residents. While current thinking tends to contradict early conclusions about the value of "edge effects" [1], it is clear that edges exert predictable forces on the habitat that have the potential to significantly alter it [2].

Hypotheses reflective of edge effects on population genetic structure are scarce [3] but may be derived from larger-scale ecological models. In forests, seed from edge species may continually intrude into the interior, eventually displacing core species $[1,4]$. Aquatic invertebrate species become less diverse along a gradient from edge to interior; in this way the edge acts as a sink for species sourced from outside the habitat fragment [5]. If genetic diversity is substituted for species diversity then one should expect it to be greatest at edges, where it can benefit from gene flow. Such a hypothesis suggests that edge-influenced population structure may appear conspicuous, if investigated.

Marine environments, especially intertidal seagrass beds (i.e., mudflats inhabited by seagrass), differ from nonmarine environments [6]. Physical aspects of the marine environment (e.g., water as a propagule vector) may reduce typical avenues of gene flow [7-9], so that genetic structure may be easily established in seagrass populations occupying heavily fragmented habitats. Physical fragmentation of beds also creates peripheral zones ("edges") that may be subject to different population-shaping forces (e.g., mortality, gap formation, propagule immigration, etc.) than interior zones [10]. Biomass at the edge of a habitat may act as a buffer, reducing the force of water flow and mixing in the interior zone [11]. As a result, edge habitat may receive more incoming reproductive propagules, and edge disturbances may cause more mortality and turnover of individuals. On the other hand, regular submersion of the entire bed during high tide events 
may constrain the effects of such buffering. The genetic structure of seagrass populations is likely to reflect these disparate forces. Microsatellite analyses of seagrass populations have demonstrated widely varying genetic diversity between populations $[12,13]$, but it is clear that populations are not the homogenous assemblages of clonal ramets that they were perceived to be [14-17].

Zostera marina L. (Zosteraceae; marine eelgrass) relies on clonal growth and sexual reproduction $[18,19]$. Clonality in aquatic environments may be a response to the uncertainty of pollination, or a means to exploit stable environments [7]. Clonal populations that lack effective sexual recruitment may still consist of more than just one genet [20] and the extent of clonal spread can be large [15, 21]. Sexual reproduction contributes to genetic diversity and is known to influence the structure of $Z$. marina populations [22]. In such monoecious species, the spatial aggregation of clones creates a pollination environment that encourages geitonogamy [23] by diminishing the size of an individual's pollen shadow and making it less likely that pollen disperses beyond the boundary of the genet. These complex interactions of clonal spread and limited dispersal of sexual propagules may be reflected in patterns of heterozygosity that form at neutral loci, ostensibly due to inbreeding.

This study surveys the genetic structure of a $Z$. marina population located in the eastern Pacific Ocean at Humboldt Bay, California. The physical structure of fragmented eelgrass habitat in the bay presents an ideal opportunity to investigate the genetic effect such landscape structure can have on a plant population. Hypothetically, genetic differentiation should closely follow physical structure. Genetic diversity within a fragment reflects local clonal diversity, per longstanding assumptions regarding clonal organisms, while genetic diversity between fragments is a function of gene flow. Further, edge effects pertaining to reduced fluid mixing may hamper reproductive function, especially outcrossing, in the interior of fragments. Following this is the hypothesis that there will be greater homozygosity and clonality in the interior compared to the edge of fragments. To evaluate these hypotheses, microsatellite markers will be used to assess genetic diversity, heterozygosity, and molecular variance of the population at different spatial scales.

\section{Materials and Methods}

2.1. Study System. Zostera marina is a common seagrass throughout the northern hemisphere. It is typically found in sheltered estuarine embayments [18]. Individuals of the species are monoecious, bearing imperfect flowers of both sexes together on long, flattened inflorescences. Flowers of Z. marina exhibit protogyny; female flowers conclude their phase of pollen receptivity at least 24 hours before the initiation of anther dehiscence [24]. The species is self-compatible [25]. Z. marina populations can be either annual or perennial, depending on local environmental conditions. Annual populations are most likely encountered in relatively unsheltered habitats, or at the north latitudinal extreme of the species range, where harsh seasonal conditions, such as

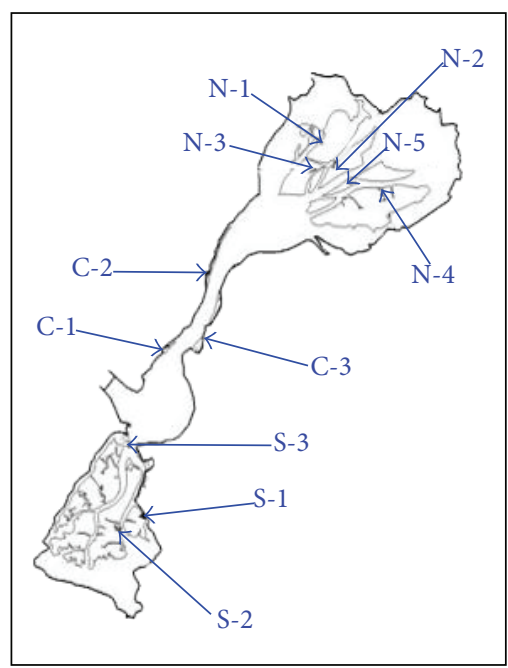

Figure 1: Map of Humboldt Bay showing plot locations. Eelgrass beds are outlined in gray.

freezing, reduce the survival of eelgrass $[18,22]$. Perenniality is typically achieved through rhizomatous growth, since above-ground shoots tend to deteriorate over the course of a growing season in the near-shore habitat. The rhizomatous habit also makes clonal replication likely. Z. marina habitats vary, from shallow subtidal to intertidal, where brief, regular periods of exposure to a terrestrial environment occur.

Humboldt Bay is located on the north coast of California, USA, and supports a large Zostera marina population. Individuals at Humboldt Bay produce flowering shoots as well as rhizomes, suggesting that the local population is maintained by a combination of sexual reproduction and clonal growth. Eelgrass habitat in the interior of the bay is subdivided into distinct intertidal mudflats bounded by dredged channels that provide relatively little subtidal habitat. Habitat around the periphery of the bay consists of gradually sloping beaches, providing intertidal and subtidal habitat. Dredging has shaped most of the eelgrass habitat into isolated mudflat beds with discrete edges that are exposed during some low tides. Spread of a genet between these habitat sites is unlikely since there is no medium for rhizome growth present, and establishment of eelgrass from vegetative propagules has a low probability of success [26].

2.2. Specimen Collection. Fresh plant material was collected at low tide periods during June 2003. Sampling was conducted in eleven different eelgrass beds (Figure 1). Five beds were sampled in the northern portion of the bay, while three beds were sampled in each of the central and southern portions. Sampling did not occur in any areas of restored eelgrass habitat. Sampling was conducted within one $20 \mathrm{~m} \times 40 \mathrm{~m}$ plot per bed. The location of each plot was determined such that one narrow side was placed adjacent to the low tide boundary of the bed (the edge) while all other sides were at least $3 \mathrm{~m}$ distant from the edge of the bed (i.e., the beds in which the plots were placed were larger than the plots themselves). Each plot had five sampling points, two of which 
were placed at random intervals along the bed edge and three located at random locations in the interior (more than $1 \mathrm{~m}$ from the edge) of the plot. At each sampling point, up to nine leaves were collected from different arbitrarily chosen eelgrass ramets in a $1 \mathrm{~m}$ radius. The specimens were coated in silica gel crystals and then stored at $-20^{\circ} \mathrm{C}$.

2.3. Laboratory Procedure. Extraction of DNA for microsatellite analysis was conducted with the aid of a Promega Wizard Genomic DNA purification kit. One extraction was performed for each ramet collected. Extracted DNA in buffer solution was analyzed for DNA concentration using a spectrophotometer and then diluted to a standard of $50 \mathrm{ng} / \mu \mathrm{L}$. These working samples were frozen at $-20^{\circ} \mathrm{C}$ until needed for PCR.

ZOSMAR primers [16, 27] were selected for use based on their potential to yield polymorphic results when applied to the Humboldt Bay gene pool. A test of primers provided by the Molecular Ecology Laboratory, Alaska Science Center, revealed four highly polymorphic loci: CT-12, CT-20, GA-2, and GA-3. These loci were deemed sufficient to characterize the population sample in an unbiased manner, based on the need to reject or accept clonal identity. The likelihood of independently sampling genetically identical genets was estimated $P=0.00369$, sensu Parker et al. [28]. At that rate the misclassification of nonclones as clones, or outbreeding as inbreeding, was unlikely; the remote possibility of such error made estimates of genetic diversity and heterozygosity slightly conservative.

Samples were prepared for PCR by combining reagents in a 96-well thermocycler plate for a Techne Genius thermocycler. Each well of a plate contained $2.95 \mu \mathrm{L}$ salt/buffer solution, $1.0 \mu \mathrm{L}$ DNA sample, $0.5 \mu \mathrm{L}$ of each forward and reverse primer to be used, and $0.05 \mu \mathrm{L}$ Taq. The CT-12 and CT-20 primers were labeled with VIC and the GA-2 and GA-3 primers were labeled with NED fluorescent labels, to allow multiplexing of primers within wells. Microsatellite sequences were taken from published records $[16,27]$. Once a plate was prepared, it was immediately loaded into the thermocycler and processed using the following cycles, durations, and temperatures: one cycle at $94^{\circ} \mathrm{C}$ for $2 \mathrm{~min}, 40$ cycles at $94^{\circ} \mathrm{C}$ for $15 \mathrm{sec} / 50^{\circ} \mathrm{C}$ for $15 \mathrm{sec} / 72^{\circ} \mathrm{C}$ for $30 \mathrm{sec}$, and one cycle at $72^{\circ} \mathrm{C}$ $30 \mathrm{~min}$. Upon completion of PCR, the plate was refrigerated at $5^{\circ} \mathrm{C}$ until the samples were needed for genotyping.

Samples of amplified DNA were prepared for genotyping by transferring a $0.5 \mu \mathrm{L}$ subsample to a new 96-well plate where the wells had been loaded with a mixture of $10.5 \mu \mathrm{L}$ formamide and $0.5 \mu \mathrm{L} \mathrm{LIZ} 500$ size-standard. Once a plate was prepared for genotyping it was processed at an on-site sequencing facility, where it was stored at $5^{\circ} \mathrm{C}$ until it was analyzed by an ABI 3730 DNA analyzer that night.

2.4. Data Analysis. Data output from the sequencer was visualized using ABI Prism Genemapper 3.5 software. All genotypes were manually scored for base pair length. Once all individuals were genotyped, scored "alleles" differing by a small number of base pairs were grouped into a single allelic class. This approach assumed some error in the level of accuracy of sequencing, since assignment of a unique allele to every base pair length reported would have resulted in a high and unlikely number of unique alleles. Analysis was of the full data set (469 individuals) and of a "trimmed" data set (360 individuals) in which individuals lacking unequivocal multilocus genotypes were removed.

Two indices of genetic diversity were computed for each sampling plot, as well as for edge and interior samples. The first (referred to henceforth as "Ellstrand and Roose's"), which computes diversity (sometimes identified as the variable " $C$ " or " $P_{d}$ " in literature) as the number of genets divided by the number of ramets [20], has previously been used to compute genetic diversity in eelgrass populations $[13,29,30]$. Because this method does not account for relative frequency of genotypes (i.e., a particular genotype with redundant clones is only counted once), Simpson's diversity index (sometimes identified as " $D$ " in literature) was also computed for the same data. Simpson's diversity index [31] computes the relative frequency of each genotype present and then sums the results for a final value indicating total diversity. One-way analyses of variance were used to compare diversity measures in the 11 beds included in the survey. Variance between positions within a bed (edge or interior) was assessed using a nested ANOVA in which bed position was used as the main effect and each diversity index served as a response variable. Analyses were conducted using the JMP IN 5.1.2 software package [32].

Data were also analyzed for deviation from HardyWeinberg equilibrium (HWE) and analysis of molecular variance (AMOVA) using ARLEQUIN version 2.0 [33]. For the HWE analysis, the full data set was grouped into separate beds as well as edge and interior samples. These sample sets varied in the number of observations included in each. Computations for HWE used default settings (100,000 steps in Markov chain; 1000 dememorization steps). To assure experimentwise error rate of 0.05 , Bonferroni-corrected comparisonwise alpha $(\alpha)$ values were used to determine significant differences.

Two types of AMOVA were performed using sampling point data to compose ARLEQUIN samples. In the first AMOVA, the data set was organized for analysis of differentiation between plots. Data collected at each point were placed in separate ARLEQUIN samples, for a total of 54 samples. These were further grouped by plot. In the second AMOVA, the data set was organized for analysis of differentiation between bed positions. Both AMOVAs used the trimmed data set, to prevent "incomplete" data for entire loci from being discarded by the software.

\section{Results and Discussion}

3.1. Genetic Characterization. The microsatellite loci used in this study were all polymorphic with six to eight alleles each (Table 1) and showed a medium to high amount of allelic diversity for an eastern Pacific population of Zostera marina $[17,29]$. For each locus, there were one or two alleles and genotypes that occurred much more frequently than the others. 
TABLE 1: Number of observed alleles and frequencies of most common allele and genotype for each locus.

\begin{tabular}{lccc}
\hline Locus & Number of alleles & Allele frequency & $\begin{array}{c}\text { Genotype } \\
\text { frequency }\end{array}$ \\
\hline CT-12 & 8 & 0.767 & 0.595 \\
CT-20 & 8 & 0.504 & 0.523 \\
GA-2 & 6 & 0.610 & 0.407 \\
GA-3 & 6 & 0.802 & 0.638 \\
\hline
\end{tabular}

Mean genetic diversity varied widely among plots and less so among bed positions (Table 2). Ellstrand and Roose's diversity index $[20]$ resulted in significantly different means $(P=$ 0.0248 ) among plots. There were no significant differences between bed positions using Ellstrand and Roose's index, or for Simpson's diversity index [31] using either grouping. The surprisingly high mean values of genetic diversity at the plot level of analysis suggest that the spread of clonal genets is limited. This reinforces conclusions elsewhere $[13,17]$ that clonality is not as pervasive as once thought. Relative frequency of genotypes varies widely in the data, with few common types and many scarce types. Results of Simpson's diversity index show that genetic diversity in Humboldt Bay does not differ among beds. Some physical variation exists around the bay (e.g., habitats found in the south part of the bay are marginally higher in elevation than those in the north part of the bay), but such differences do not appear to influence the extent of clonality in Humboldt Bay.

Comparisons of observed $\left(H_{O}\right)$ and expected $\left(H_{E}\right)$ heterozygosity showed few departures from HWE throughout the population (Table 3 ). Unequal sample sizes likely contribute to differences in the ability to detect departure from HWE. Deficits in heterozygosity may result from nonrandom mating (i.e., higher incidences of self-pollination result in more homozygotes). The clonal nature of eelgrass makes geitonogamous selfing likely at finer scales, where a ramet may be in the presence of sister ramets, and the limited dispersal ability of sexual propagules makes escaping the spread of one's own genet uncertain $[8,9,34]$. Genetic drift, due to small population size, could also cause deviation from HWE but genetic drift is unlikely because results of AMOVA suggest effective gene flow between beds [35] and hence large effective population size. Factors contributing to excess heterozygotes are less clear but may include gene flow from other populations [6]. Plot Central-1, which exhibits excess heterozygosity at one locus, is positioned favorably to intercept rafting shoots from other populations (Figure 1). Such a hypothesis is highly speculative, since there is no information available on the rate or likelihood of rafting shoot arrival from other populations.

3.2. Population Structure. Table 4 presents the full results of the AMOVA for each data set. In the data set in which samples were grouped by plot, most of the detectable variation (approx. 84\%) occurs within sampling points (i.e., among the nine individuals sampled per point). Variation within plots is approximately $15 \%$ of the total. Variation between sampling plots accounted for less than one percent of the total. These
TABLE 2: Mean values \pm standard errors of genetic diversity indices; by plot, bed position, and the entire population; $P$ values from tests of significance of genetic diversity. Significance at the level of $\alpha=$ 0.05 is indicated by “*”.

\begin{tabular}{lcc}
\hline Plot & Ellstrand and Roose $(C)$ & Simpson $(D)$ \\
\hline S-1 & $0.67 \pm 0.09$ & $0.96 \pm 0.07$ \\
S-2 & $0.58 \pm 0.06$ & $0.92 \pm 0.03$ \\
S-3 & $0.67 \pm 0.11$ & $0.94 \pm 0.14$ \\
C-1 & $0.79 \pm 0.09$ & $0.96 \pm 0.04$ \\
C-2 & $0.57 \pm 0.12$ & $0.94 \pm 0.09$ \\
C-3 & $0.82 \pm 0.08$ & $0.96 \pm 0.09$ \\
N-1 & $0.34 \pm 0.18$ & $0.85 \pm 0.20$ \\
N-2 & $0.64 \pm 0.11$ & $0.92 \pm 0.09$ \\
N-3 & $0.47 \pm 0.10$ & $0.94 \pm 0.07$ \\
N-4 & $0.83 \pm 0.07$ & $0.97 \pm 0.03$ \\
N-5 & $0.44 \pm 0.05$ & $0.94 \pm 0.11$ \\
\hline Edge & $0.63 \pm 0.06$ & $0.75 \pm 0.06$ \\
Interior & $0.62 \pm 0.05$ & $0.77 \pm 0.04$ \\
\hline Population & $0.62 \pm 0.04$ & $0.76 \pm 0.03$ \\
\hline Tests of significance & & \\
\multicolumn{1}{c}{ Among plots } & $0.0248^{*}$ & 0.1126 \\
Among bed positions & 0.8603 & 0.7175 \\
\hline
\end{tabular}

results suggest that the nearest neighbors, within $1 \mathrm{~m}$ radius, are rarely clones. Presumably most sampled individuals at that scale either originated from seed or genets have become highly interdigitated through growth. Less pronounced variability within plots may lead to speculation that large clones and/or short-range propagule dispersal contribute to homogenization of variation at that scale. Perhaps most surprising is, considering the fragmented nature of the beds, the negligible variation observed between plots. Such a result presents a picture of widespread homogeneity throughout the bay, excluding the argument that each bed is a population unto itself (and the bay a metapopulation). One may speculate that mechanisms such as effective propagule dispersal and persistence of ancient clones contribute to such a pattern. At such a scale, rafting reproductive shoots may play a role in the projection of propagules beyond their limited ranges when moved singly [36]. Genets have been described as potentially very long-lived [37] and the time since the installation of most channels is only on the order of decades, leaving open the possibility that prechannel clones remain in multiple fragments. The historical presence of such far-spanning genets would suggest that the role of clonal growth in the population has shifted significantly over time.

The bed position data set shows that the amount of variation found within and between sampling points is very similar to the plot data set. Very little variation $(0.52 \%)$ is detected between edge and interior regions. When considered alongside the similar genetic diversity measures (Table 2) and inconsistent deviations from HWE (Table 3), these suggest that reproductive strategies between edge and interior positions do not differ in any meaningful way. Since edges and 
TABLE 3: Values of observed and expected heterozygosity at four microsatellite loci. Data is arranged by plot and bed position. Multiply sampled multilocus genotypes were excluded. Significant $P$ values are denoted by “*” which indicates a departure from Hardy-Weinberg equilibrium. To assure experimentwise error rate of 0.05 , Bonferroni-corrected comparisonwise alpha values of (a) 0.00114 and (b) 0.00625 were used.

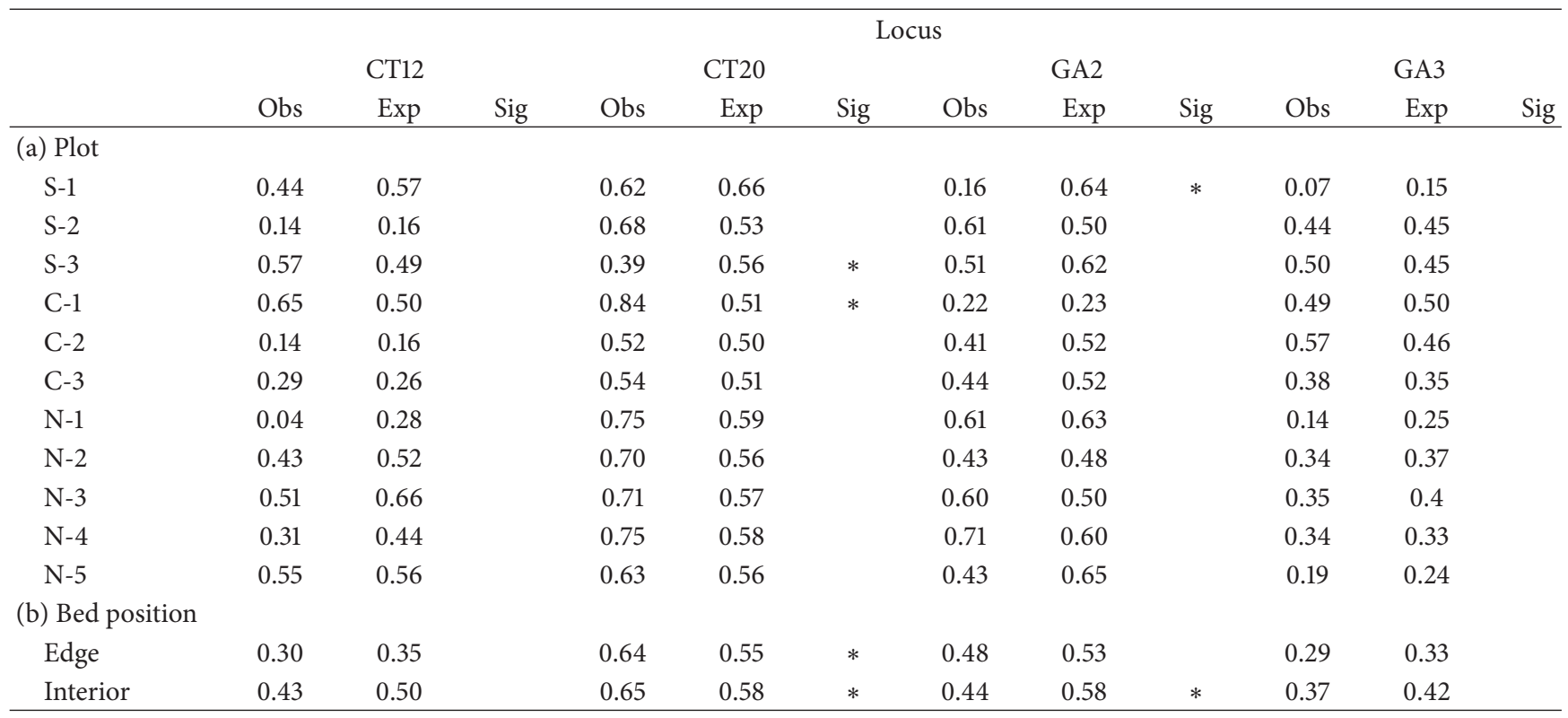

TABLE 4: AMOVA results for the plot and bed position data sets. Multiply sampled multilocus genotypes were excluded. The majority of the total genetic variation shown in both tables occurs at the finest scale included in the analysis ("points").

\begin{tabular}{lcccc}
\hline Source of variation & DF & Sum of squares & Variation components & Percent of variation \\
\hline Among plots & 10 & 16.63 & 0.004 & 0.83 \\
Among points within plots & 43 & 56.46 & 0.071 & 15.29 \\
Within points & 670 & 259.46 & 0.387 & 83.88 \\
Total & $\mathbf{7 2 3}$ & $\mathbf{3 3 2 . 5 4}$ & $\mathbf{0 . 4 6 2}$ & 0.00242 \\
\hline Among bed positions & 1 & 2.339 & 0.07292 & 15.76 \\
Among points within bed positions & 52 & 70.746 & 0.38725 & 83.71 \\
Within points & 670 & 259.455 & $\mathbf{0 . 4 6 2 5 8}$ & \\
Total & $\mathbf{7 2 3}$ & $\mathbf{3 3 2 . 5 4 0}$ & & \\
\hline
\end{tabular}

interiors are adjacent, clonal spread of genets between positions could account for at least part of the homogenization observed at that scale. Also, the frequent submergence of beds during high tides could reasonably be expected to exert a constraining effect on selection of reproductive strategies due to a weakened effect of physical differences (i.e., by forming a uniform edge at the water's surface to which edge and interior ramets would be subjected equally) $[11,38]$.

In analyses of microsatellite loci, awareness of the effect null alleles may have upon results is worthwhile [39]. Like most foundational studies on eelgrass genetics [13, 29, 40, 41], an estimate of null alleles was not employed here. In one study that did estimate the effect of null alleles, Campanella et al. [30] found little influence by null alleles upon computed $F$-statistics. Accordingly, explorations of null allele effects have revealed little or no bias from null alleles in genetically unstructured populations [39], like that under study here. Even if null alleles were present, they would result in false positive identification of homozygote genotypes, thus making estimates of heterozygosity and outcrossing more conservative.

\section{Conclusions}

Hypotheses inspired by principles of clonal plant reproduction and ecological edge effects were tested in this mensurative field study of eelgrass genetics in Humboldt Bay. Analysis of four polymorphic loci showed infrequent deviations from HWE, little difference in genetic diversity across the bay, and most genetic variation present at the finest scale analyzed. A prediction of distinct genetic differences between eelgrass beds, where different clones would presumably grow to dominate the physically isolated habitat fragments, was rejected by a careful analysis of key population genetic metrics at different spatial scales. Similarly, comparisons between edge and 
interior samples rejected a prediction that inbreeding and clonal dominance would be more likely away from fragment edges. Tidal cycles may moderate differences that might otherwise occur between edge and interior positions in a bed.

Seagrass stands are typically limited to one or a few plant species [42], which could undermine their perceived conservation value when compared to relatively species-rich habitats. Measures of genetic diversity offer an alternative for the assessment of conservation value in seagrass habitats [43]; results provided here closely match a growing trend in which eelgrass populations are shown to be more genetically diverse than anticipated $[17,29,41]$. Two similar studies $[40,44]$ empirically tested the role of genetic diversity in a seagrass population's ability to recover from disturbances. They find that the time needed for a population to recover is less with increasing genetic diversity, and that, in addition to increased production of seagrass biomass, increased abundance is also seen in other members of the community. The results presented here may be useful to natural resource managers of Humboldt Bay and similar temperate zone estuaries after disturbance events such as high temperatures, herbivore activities, or disease affect the eelgrass population located there. Specifically, variation in the recovery of eelgrass at different locations in the bay may be attributed to factors other than genetic diversity, since genetic diversity was not found to appreciably differ among bay locations.

\section{Conflict of Interests}

The author declares that there is no conflict of interests regarding the publication of this paper.

\section{Acknowledgments}

Thanks are due to J. Riggs, F. Shaughnessy, S. Ferson, S. Schlosser, N. Kalson, V. Frey, J. Robinson, and G. Tennant for logistical and conceptual assistance at the study site. Thanks are due to A. Ballard, S. Talbot, K. Sage, and D. Pouchnik for instruction and advice working with microsatellites. Thanks are due to M. Morgan, J. Bishop, L. Hufford, C. Omoto, N. Palmeri, and one anonymous reviewer for helpful comments on the paper. B. Balk provided valuable assistance formatting Figure 1. This work was funded in part by the Betty W. Higinbotham research fellowship to Joshua S. Neely and NSF Grant 0128896 to M. Morgan. This work represents a portion of the dissertation submitted by Joshua S. Neely to Washington State University, entitled "Reproduction and adaptation in eastern Pacific eelgrass populations."

\section{References}

[1] D. S. Wilcove, C. H. McLellan, and A. P. Dobson, "Habitat fragmentation in the temperate zone," in Conservation Biology: the Science of Scarcity and Diversity, pp. 237-256, Sunderland, Sinauer Associates, 1986.

[2] L. Ries, R. J. Fletcher Jr., J. Battin, and T. D. Sisk, "Ecological responses to habitat edges: mechanisms, models, and variability explained," Annual Review of Ecology, Evolution, and Systematics, vol. 35, pp. 491-522, 2004.
[3] L. Fahrig, "Effects of habitat fragmentation on biodiversity," Annual Review of Ecology, Evolution, and Systematics, vol. 34, pp. 487-515, 2003.

[4] J. W. Ranney, M. C. Brunner, and J. B. Levenson, "The importance of edge in the structure and dynamics of forest islands," in Forest Island Dynamics in Man-Dominated Landscapes, pp. 67-96, Springer, New York, NY, USA, 1981.

[5] R. M. Ewers and R. K. Didham, "Confounding factors in the detection of species responses to habitat fragmentation," Biological Reviews of the Cambridge Philosophical Society, vol. 81, no. 1, pp. 117-142, 2006.

[6] R. H. Laushman, "Population genetics of hydrophilous angiosperms," Aquatic Botany, vol. 44, no. 2-3, pp. 147-158, 1993.

[7] D. H. Les, "Breeding systems, population structure, and evolution in hydrophilous angiosperms," Annals of the Missouri Botanical Garden, vol. 75, pp. 819-835, 1988.

[8] R. J. Orth, M. Luckenbach, and K. A. Moore, "Seed dispersal in a marine macrophyte: implications for colonization and restoration," Ecology, vol. 75, no. 7, pp. 1927-1939, 1994.

[9] M. H. Ruckelshaus, "Estimation of genetic neighborhood parameters from pollen and seed dispersal in the marine angiosperm Zostera marina L," Evolution, vol. 50, no. 2, pp. 856-864, 1996.

[10] K. A. Hovel and R. N. Lipcius, "Habitat fragmentation in a seagrass landscape: patch size and complexity control blue crab survival," Ecology, vol. 82, no. 7, pp. 1814-1829, 2001.

[11] J. D. Ackerman and A. Okubo, "Reduced mixing in a marine macrophyte canopy," Functional Ecology, vol. 7, no. 3, pp. 305309, 1993.

[12] G. Procaccini, M. V. Ruggiero, and L. Orsini, “Genetic structure and distribution of microsatellite population genetic diversity in Posidonia oceanica in the Mediterranean basin," Bulletin of Marine Science, vol. 71, no. 3, pp. 1291-1297, 2002.

[13] T. B. H. Reusch, W. T. Stam, and J. L. Olsen, "A microsatellitebased estimation of clonal diversity and population subdivision in Zostera marina, a marine flowering plant," Molecular Ecology, vol. 9, no. 2, pp. 127-140, 2000.

[14] T. B. H. Reusch, "New markers-old questions: population genetics of seagrasses," Marine Ecology Progress Series, vol. 211, pp. 261-274, 2001.

[15] S. C. H. Barrett, C. G. Eckert, and B. C. Husband, "Evolutionary processes in aquatic plant populations," Aquatic Botany, vol. 44, no. 2-3, pp. 105-145, 1993.

[16] T. B. H. Reusch, W. T. Stam, and J. L. Olsen, "Microsatellite loci in eelgrass Zostera marina reveal marked polymorphism within and among populations," Molecular Ecology, vol. 8, no. 2, pp. 317-321, 1999.

[17] R. Muñiz-Salazar, S. L. Talbot, G. K. Sage, D. H. Ward, and A. Cabello-Pasini, "Genetic structure of eelgrass Zostera marina meadows in an embayment with restricted water flow," Marine Ecology Progress Series, vol. 309, pp. 107-116, 2006.

[18] C. Den Hartog, The Seagrasses of the World, North Holland, Amsterdam, The Netherlands, 1970.

[19] C. D. Sculthorpe, The Biology of Aquatic Vascular Plants, St. Martin's Press, New York, NY, USA, 1967.

[20] N. C. Ellstrand and M. L. Roose, "Patterns of genotypic diversity in clonal plant species," The American Journal of Botany, vol. 74, no. 1, pp. 123-131, 1987.

[21] J. B. Grace, "The adaptive significance of clonal reproduction in angiosperms: an aquatic perspective," Aquatic Botany, vol. 44, no. 2-3, pp. 159-180, 1993. 
[22] T. B. H. Reusch, "Pollination in the marine realm: microsatellites reveal high outcrossing rates and multiple paternity in eelgrass Zostera marina," Heredity, vol. 85, no. 5, pp. 459-464, 2000.

[23] S. N. Handel, "The intrusion of clonal growth patterns on plant breeding systems," The American Naturalist, vol. 125, no. 3, pp. 367-384, 1985.

[24] A. W. A. M. de Cock, "Flowering, pollination and fruiting in Zostera marina L," Aquatic Botany, vol. 9, pp. 201-220, 1980.

[25] M. H. Ruckelshaus, "Estimates of outcrossing rates and of inbreeding depression in a population of the marine angiosperm Zostera marina," Marine Biology, vol. 123, no. 3, pp. 583-593, 1995.

[26] P. J. Ewanchuk and S. L. Williams, "Survival and re-establishment of vegetative fragments of eelgrass (Zostera marina)," Canadian Journal of Botany, vol. 74, no. 10, pp. 1584-1590, 1996.

[27] T. B. H. Reusch, "Five microsatellite loci in eelgrass Zostera marina and a test of cross-species amplification in Z. noltii and Z. japonica," Molecular Ecology, vol. 9, no. 3, pp. 371-373, 2000.

[28] P. G. Parker, A. A. Snow, M. D. Schug, G. C. Booton, and P. A. Fuerst, "What molecules can tell us about populations: choosing and using a molecular marker," Ecology, vol. 79, no. 2, pp. 361$382,1998$.

[29] J. L. Olsen, W. T. Stam, J. A. Coyer et al., "North Atlantic phylogeography and large-scale population differentiation of the seagrass Zostera marina L," Molecular Ecology, vol. 13, no. 7, pp. 1923-1941, 2004.

[30] J. J. Campanella, P. A. Bologna, J. V. Smalley, E. B. Rosenzweig, and S. M. Smith, "Population structure of Zostera marina (Eelgrass) on the Western Atlantic Coast is characterized by poor connectivity and inbreeding," Journal of Heredity, vol. 101, no. 1, pp. 61-70, 2010.

[31] E. H. Simpson, "Measurement of diversity," Nature, vol. 163, p. 688, 1949.

[32] J. Sall, L. Creighton, and A. Lehman, JMP IN 5.1, SAS Institute, 2003.

[33] S. Schneider, D. Roessli, and L. Excoffier, Arlequin Version 2.000: A Software for Population Genetics Analysis, Genetics and Biometry Laboratory, University of Geneva, Geneva, Switzerland, 2000.

[34] T. B. H. Reusch, "Fitness-consequences of geitonogamous selfing in a clonal marine angiosperm (Zostera marina)," Journal of Evolutionary Biology, vol. 14, no. 1, pp. 129-138, 2001.

[35] M. Slatkin, "Gene flow and the geographic structure of natural populations," Science, vol. 236, no. 4803, pp. 787-792, 1987.

[36] T. B. H. Reusch, "Microsatellites reveal high population connectivity in eelgrass (Zostera marina) in two contrasting coastal areas," Limnology and Oceanography, vol. 47, no. 1, pp. 78-85, 2002.

[37] O. Eriksson, "Dynamics of genets in clonal plants," Trends in Ecology \& Evolution, vol. 8, no. 9, pp. 313-316, 1993.

[38] J. D. Ackerman, "Diffusivity in a marine macrophyte canopy: implications for submarine pollination and dispersal," American Journal of Botany, vol. 89, no. 7, pp. 1119-1127, 2002.

[39] M.-P. Chapuis and A. Estoup, "Microsatellite null alleles and estimation of population differentiation," Molecular Biology and Evolution, vol. 24, no. 3, pp. 621-631, 2007.

[40] A. R. Hughes and J. J. Stachowicz, "Genetic diversity enhances the resistance of a seagrass ecosystem to disturbance," Proceedings of the National Academy of Sciences of the United States of America, vol. 101, no. 24, pp. 8998-9002, 2004.
[41] R. Muñiz-Salazar, S. L. Talbot, G. K. Sage, D. H. Ward, and A. Cabello-Pasini, "Population genetic structure of annual and perennial populations of Zostera marina L. along the Pacific coast of Baja California and the Gulf of California," Molecular Ecology, vol. 14, no. 3, pp. 711-722, 2005.

[42] C. M. Duarte, “The future of seagrass meadows," Environmental Conservation, vol. 29, no. 2, pp. 192-206, 2002.

[43] R. J. Orth, T. J. B. Carruthers, W. C. Dennison et al., "A global crisis for seagrass ecosystems," BioScience, vol. 56, no. 12, pp. 987-996, 2006.

[44] T. B. H. Reusch, A. Ehlers, A. Hämmerli, and B. Worm, "Ecosystem recovery after climatic extremes enhanced by genotypic diversity," Proceedings of the National Academy of Sciences of the United States of America, vol. 102, no. 8, pp. 2826-2831, 2005. 

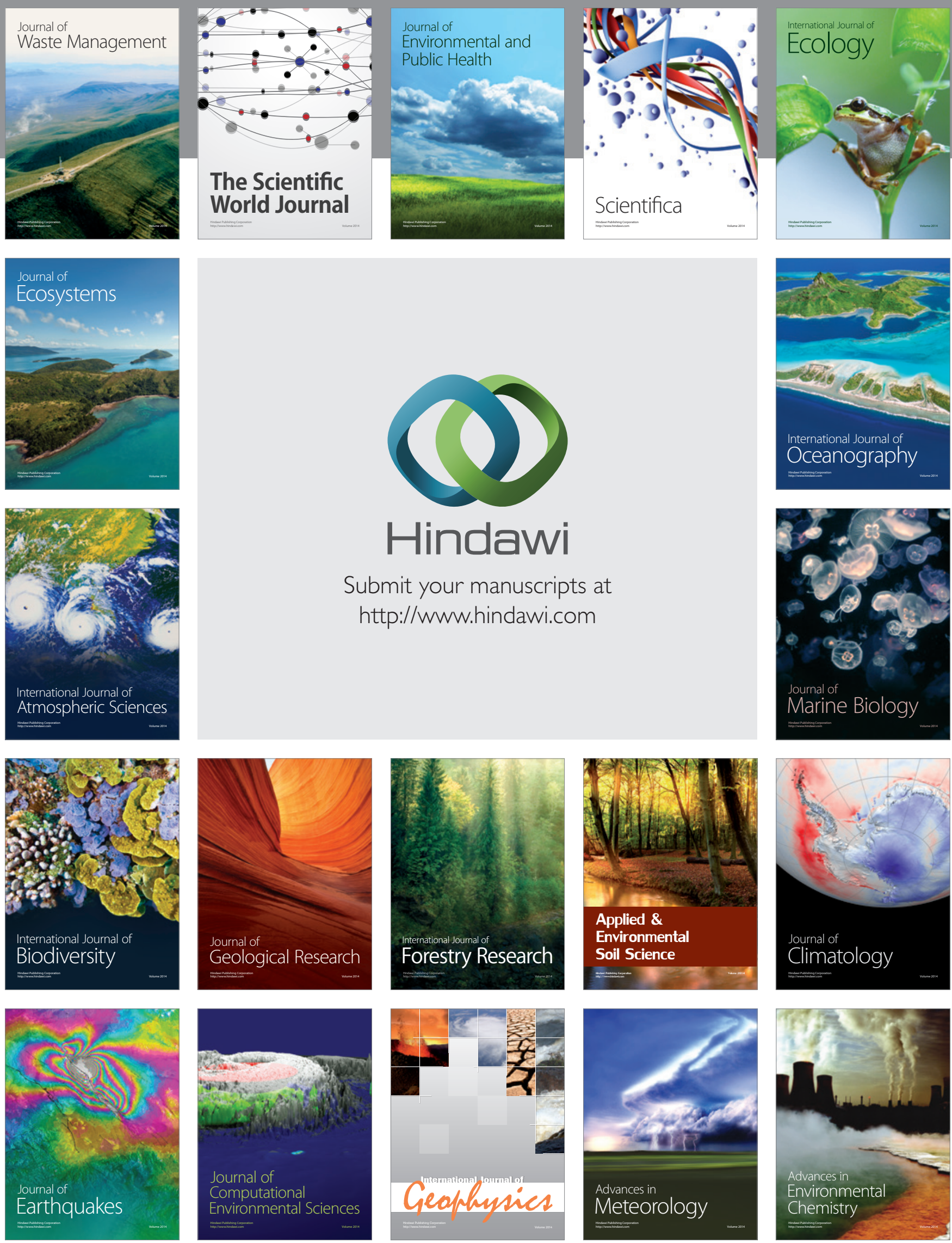